\title{
Optical absorption from graphitic clusters of hydrogenated amorphous carbon thin films
}

\author{
E. Pascual, C. Serra, and E. Bertran \\ Departament de Fisica Aplicada i Electrònica, Universitat de Barcelona, Av. Diagonal 645-647, \\ E08028-Barcelona, Spain
}

(Received 24 April 1991; accepted for publication 29 July 1991)

\begin{abstract}
The optical absorption of hydrogenated amorphous carbon films ( $a-\mathrm{C}: \mathrm{H}$ ) was measured by spectroscopic ellipsometry. The $a-\mathrm{C}: \mathrm{H}$ films were deposited at different substrate temperatures by rf-plasma of methane. A volume distribution of graphitic cluster size was assumed to reproduce the experimental spectra of the absorption coefficient. The changes in the absorption coefficient and the optical gap, induced by deposition temperature, have been interpreted in terms of changes in the graphitic cluster size of the network.

The increase in the deposition temperature produces an increase in the size of the graphitic clusters.
\end{abstract}

Among the different interpretations of the amorphous carbon microstructure, ${ }^{1-8}$ the model proposed by Robertson and $\mathrm{O}^{\prime}$ Reilly ${ }^{7,8}$ allows the interpretation of the optical measurements of the hydrogenated amorphous carbon ( $a$ $\mathrm{C:H})$ films with good agreement between theory and experiment. In this microstructural model, they considered that the most stable arrangement of $s p^{2}$ sites is in compact clusters of fused sixfold rings, which are bound by $s p^{2}$ or $s p^{3}$ hydrogen and $s p^{3}$ carbon sites.

The existence of an optical gap in hydrogenated amorphous carbon ( $a$-C:H) can be explained by $\pi$-bonded clusters showing $s p^{2}$ coordination. ${ }^{7,9,10}$ This $s p^{2}$ coordination is characteristic of the graphitic structures, which have been experimentally found by infrared (IR) analysis in $a-\mathrm{C}: \mathrm{H}$ films. ${ }^{11}$ In addition, a structure of $s p^{2}$-bonded carbon clusters surrounded by $s p^{3}$ - or $s p^{2}$-bonded hydrogen could explain the origin of the $1600 \mathrm{~cm}^{-1}$ vibrational band in resonant raman scattering analysis, which suggests the presence of clusters of different sizes. ${ }^{12-14}$ Recently, results of elastic recoil detection analysis (ERDA) of irradiated samples of $a-\mathrm{C}: \mathrm{H}$ have been interpreted in terms of graphitic clusters with different sizes ${ }^{15}$ and also, the results referring to the carbon bonding in $a-\mathrm{C}: \mathrm{H}$, studied by nuclear magnetic resonance (NMR) spectroscopy, are consistent with a structure of graphitic clusters. ${ }^{16}$

Taking into account the role of the graphitic clusters on the $a-\mathrm{C}: \mathrm{H}$, Brédas et al. ${ }^{10}$ have reached similar conclusions concerning the origins of the optical gap. In this way, the electronic properties of $a \cdot \mathrm{C}: \mathrm{H}$ films have also been explained as being governed by a structure of graphitic clusters.

The optical gap of $a-\mathrm{C}: \mathrm{H}$ is relatively low; ${ }^{8,17}$ not only is it much smaller than the direct gap of pure tetrahedrically bonded carbon $(7.3 \mathrm{eV})$, but it is also lower than the gap in the basic $\pi$ systems, ethylene and benzene $(5.8 \mathrm{eV})$. This has been attributed to the fact that the optical band gap of both $a-\mathrm{C}$ and $a-\mathrm{C}: \mathrm{H}$ depends on their degree of medium-range order, rather than just on their short-range order. ${ }^{8}$ The only explanation for optical gaps lower than $2.0 \mathrm{eV}$ is the existence of fused-ring aromatic structures, the width of the optical gap varying inversely with the $s p^{2}$ cluster size. $^{10}$

In order to verify the evolution of the optical absorption of the $a-\mathrm{C}: \mathrm{H}$ thin films, we have considered the contribution of a distribution of graphitic clusters to the absorption coefficient $\alpha$ through the changes induced by the deposition temperature $T_{S}$.

$T_{S}$ is an important parameter of the rf plasma deposition of $a-\mathrm{C}: \mathrm{H}{ }^{18}{ }^{18}$ Earlier studies pointed out the effect of the $T_{S}$ on the $s p^{2}$ and $s p^{3}$ coordination ratio and on the hydrogen content. ${ }^{3,19}$ The reported effects indicate that the $a$ C:H films obtained at high $T_{S}$ show low refractive index $n$ and high absorption coefficient $\alpha .{ }^{3,18,20}$

In order to study the optical absorption of $a-\mathrm{C}: \mathrm{H}$, a series of films was prepared by if glow discharge decomposition of methane ${ }^{21}$ at different substrate temperatures in the range from 20 to $400^{\circ} \mathrm{C}$. The technological conditions during the deposition process were: $60 \mathrm{~W}$ of rf power, 2.5 $\mathrm{Pa}$ of pressure, and $10 \mathrm{sccm}$ of flow rate.

The optical spectra of the $a$-C:H thin films were obtained by a spectroscopic rotating analyzer ellipsometer (RAE) in the range $1.5-4.5 \mathrm{eV} .{ }^{18,22}$ The system is controlled by a computer which calibrates the instrument following the residual method proposed by Aspnes. ${ }^{23}$ The light beam was set at $70.02^{\circ} \pm 0.01^{\circ}$ of incidence angle. The ellipsometric angles $(\Psi, \Delta)$ were calculated from a Fourier analysis of the detected light intensity. In order to reduce the effects of electrical and mechanical noise, the measurements were averaged over 120 rotations of the analyzer, providing a precision of $\pm 0.1^{\circ}$ in the ellipsometric angles.

The absorption coefficient $\alpha$ showed Tauc's behavior, for values above $10^{4} \mathrm{~cm}^{-1,24}$ which is expressed by:

$$
\alpha=B\left(E-E_{g}\right)^{2} / E,
$$

where $E_{g}$ is the optical gap energy and $B$ is a constant which get values between $5.10^{4}$ and $9.10^{4}\left(\mathrm{~cm}^{-1} / \mathrm{eV}\right)$ for these $a-\mathrm{C}: \mathrm{H}$ films. The optical gap obtained from the Tauc expression presented values from $1.1 \mathrm{eV}$ (at $20^{\circ} \mathrm{C}$ of $T_{s}$ ) to a very low value near $0.1 \mathrm{eV}$ (at $T_{S}=370^{\circ} \mathrm{C}$ ).

In order to explain the measured absorption front dependence on energy, we have considered a size volume 
distribution of graphitic clusters in an optically transparent matrix of amorphous material. Under this assumption, the energy dependence of the optical absorption could be controlled not only by clusters of average size but rather it could be determined by the larger clusters. ${ }^{8}$

Previous optical studies have considered the contribution to the optical absorption of the larger clusters ${ }^{15}$ or an exponential distribution of different sized clusters on the network; ${ }^{25}$ this latter leads to a better agreement between experimental and calculated absorption line shapes. We have tried to apply this distribution to our series of $a-\mathrm{C}: \mathrm{H}$ films, obtaining a fit only in a special case at low substrate temperature.

However, considering a volume fraction $f_{v}^{N}$ and an absorption coefficient $\alpha_{N}(E)$ associated to each cluster with $N$ rings, the total absorption $\alpha(E)$, corresponding to the total number of clusters, will be expressed as:

$$
\alpha(E)=\sum_{N=1}^{N_{L}} f_{v}^{N} \alpha_{N}(E),
$$

$N_{L}$ being the number of rings in the larger cluster of each film. The agreement between the experimental and calculated absorption has been obtained by assuming a volume fraction of the clusters of $N$ rings as follows:

$$
f_{i}^{N}=A N e^{-N / N_{\max }}
$$

where $N_{\max }$ represents the most abundant cluster and $A$ is a constant.

In this way, in a film with graphitic clusters glued together, the optical gap is mainly determined by the size of the larger graphitic species and the first electronic excitations are completely localized on the graphitic parts. ${ }^{5,10}$ This allows us to consider:

$$
\sum_{N=1}^{N_{L}} f_{v}^{N}=1
$$

The Tauc expression corresponding to the optical absorption of each kind of cluster is:

$$
\alpha_{N}(E)=B_{N}\left(E-E_{g}\right)^{2} / E
$$

where $B_{N}$ is proportional to the oscillator strength associated to $N$ carbon rings. The gap energy can be expressed as a function of $N$ by means of the expression:

$$
E_{g}=E_{g o} / \sqrt{N}
$$

where $E_{g o}=2 \beta$ is the gap energy associated to one only ring cluster, whose theoretical value is $5.8 \mathrm{cV} .^{8,9}$

Figure 1 shows the agreement between the energy dependence of the experimental data and the calculated values of the absorption coefficient $\alpha$. The results indicate a formation of larger graphitic clusters as deposition temperature increases. The dependence of size of the larger clusters on the deposition temperature shows an exponential behavior (Fig. 2):

$$
N=N_{0} e^{T_{S} / T_{0}}+N_{1}
$$

being $N_{0}, T_{0}$, and $N_{1}$ constants.

Taking into account the number of the rings in the clusters $N_{L}$, we can quantify their size, which varies from

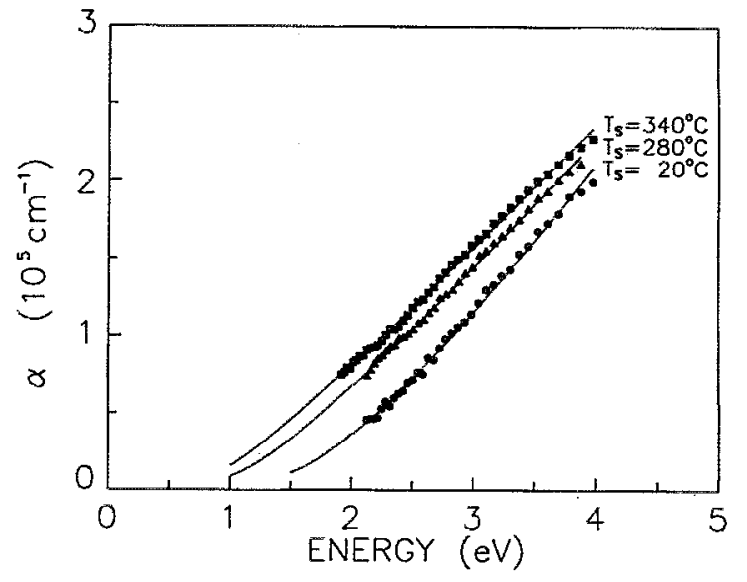

FIG. 1. Experimental absorption coefficient spectra of $a-\mathrm{C}: \mathrm{H}$ thin films grown at several deposition temperatures. Solid lines correspond to the calculated spectra assuming a volume distribution of graphitic clusters size given by $\mathrm{Eq}$. (3).

$17 \AA$ (for films grown at room temperature) to $55 \AA$ for the film obtained at $370^{\circ} \mathrm{C}$. This results supports the general idea of a graphitization process induced by an increase in the dcposition temperature. Furthermore, the number of rings in the clusters is consistent with an experimental gap in the range $0.4-0.7 \mathrm{eV}^{8}$

The calculated values of the optical gap $E_{g o}$ varied slightly for each sample, showing values around $6 \mathrm{eV}$ for all the samples grown at $T_{s}$ below $340 .{ }^{\circ} \mathrm{C}$, and decreasing towards $4 \mathrm{eV}$ for $T_{S}=370^{\circ} \mathrm{C}$ (Fig. 3). These values agree reasonably well with the theoretical value $(5.8 \mathrm{eV})$ reported by Robertson and O'Reilly. ${ }^{8}$ Considering that the gap depends primarily on the concentration and disposition of the $\pi$ states, these changes could be attributed to the different strength of the samples.

In conclusion, the analysis of the ellipsometric measurements can provide knowledge of the $a-\mathrm{C}: \mathrm{H}$ microstructure. The assumed size distribution allows the determination of the microstructural parameters of the graphitic

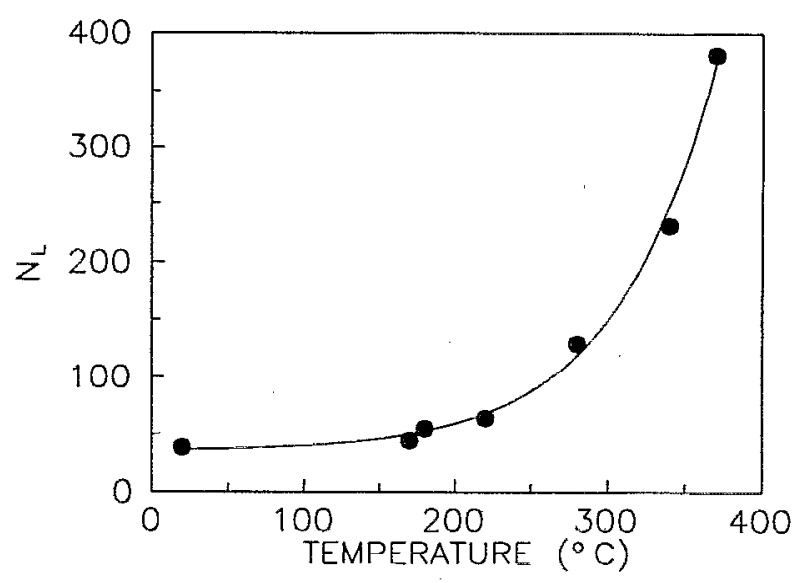

FIG. 2. Exponential behavior shown by the number of rings in the larger graphitic clusters $N_{L}$ in a-C:H thin films as a function of the deposition temperature. 


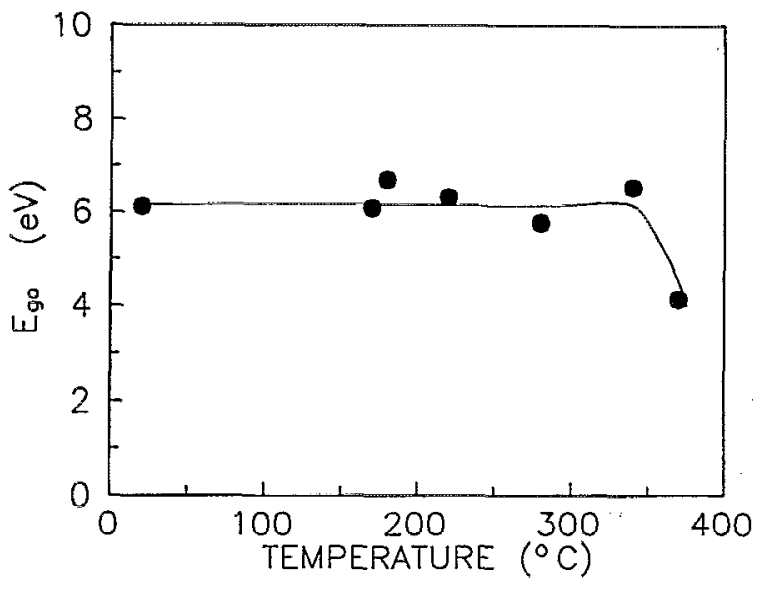

FIG. 3. Calculated values of the gap energy $E_{g o}$ from the experimental data corresponding to the $a-\mathrm{C}: \mathrm{H}$ thin films, vs deposition temperature.

clusters. The calculated clusters size yields an explanation of the low values of the optical gap, and the calculated value of $E_{g o}$ is quite consistent with the theoretical value of $5.8 \mathrm{eV}$. Finally, carbon cluster size increases exponentially with deposition temperature.

The authors are grateful to Dr. J. Esteve and F. Maass for helpful discussions. This work was financed by CICYT of Spanish Government, under contract MAT89-0221.

${ }^{1}$ J. C. Angus, P. Koidl, and S. Domitz, in Plasma Deposited Thin Films, edited by J. Mort and F. Jansen (CRC, Boca Raton, FL, 1986).

${ }^{2}$ B. Dischler, A. Bubenzer, and P. Koidl, Appl. Phys. Lett. 42, 636 (1983).

${ }^{3}$ D. A. Anderson, Philos. Mag. 35, 17 (1977).
${ }^{4}$ J. Fink, T. Müller-Heinzerling, J. Pflüger, A. Bubenzer, P. Koidl, and G. Creceliūs, Solid State Commun. 47, 687 (1983).

${ }^{5}$ D. Beeman, J. Silverman, R. Lynds, and M. R. Anderson, Phys. Rev. B 30, 870 (1984).

${ }^{6}$ C. Weissmantel, K. Bewilogua, C. Schürer, K. Breuer, and H. Zscheile, Thin Solid Films 61, L1 (1979).

${ }^{7}$ J. Robertson, 1 dv. Phys. 32, 361 (1983).

${ }^{8}$ J. Robertson and E. P. O'Reilly, Phys. Rev. B 35, 2946 (1987).

${ }^{9}$ E. P. O'Reilly, J. Robertson, and D. Beeman, J. Non-Cryst. Solids 77-78, 83 (1985).

${ }^{10}$ J. L. Brédas and G. B. Street, J. Phys. C 18, L651 (1985).

${ }^{11}$ B. Dischler, A. Bubenzer, and P. Koidl, Solid State Commun. 48, 105 (1983).

${ }_{12}^{12}$ M. Ramsteiner and J. Wagner, Appl. Phys. Lett. 51, 1355 (1987).

${ }^{13}$ I. Sela, M. Adel, and R. Beserman, J. Appl. Phys. 68, 70 (1990).

${ }^{14}$ M. Yoshikawa, G. Katagiri, H. Ishida, A. Ishitani, and T. Akamatsu, Appl. Phys. Lett. 52, 1639 (1988).

${ }^{15}$ G. Compagnini, G. Foti, R. Reitano, and G. Mondio, Appl. Phys. Lett. 57, 2546 (1990).

${ }^{16}$ M. A. Tamor, W. C. Vassell, and K. R. Carduner, Appl. Phys. Lett. 58, 592 (1991).

${ }^{17}$ S. Kaplan, F. Jansen, and M. Machonkin, Appl. Phys. Lett. 47, 750 (1985).

${ }^{18}$ E. Pascual, C. Serra, J. Esteve, and E. Bertran, Surf. Coat. Tech. 47, 263 (1991).

${ }^{19}$ K. Fabisiak, S. Oreszko, F. Rozploch, and J. Szatkowski, J. Non-Cryst. Solids 99, 12 (1988).

${ }^{20}$ K. Pirker, R. Schallauer, W. Fallmann, O. Olkaytug, G. Urban, A. Jachimowicz, F. Kohl, and O. Prohaska, Thin Solid Films 138, 121 (1986).

${ }^{21}$ C. Serra, E. Pascual, F. Maass, and J. Esteve, Surf. Coat. Tech. 47, 89 (1991).

${ }^{22}$ E. Pascual, M. C. Polo, J. Esteve, and E. Bertran, Surf. Sci. 251-252, 200 (1991).

${ }^{23}$ D. E. Aspnes and A. A. Studna, Appl. Opt. 14, 220 (1975).

${ }^{24}$ E. A. Davis and N. F. Mott, Philos. Mag. 22, 903 (1970).

${ }^{25}$ V. Barbarossa, F. Galluzzi, S. Mercuri, R. Tomaciello, and G. Grillo, International Conference on New Diamond Science and Technology, edited by R. Messier and J. Glass (Materials Research Society, Washington, DC, 1990). 